\title{
Development of intuitionistic fuzzy integrated super-efficiency SBM model
}

\author{
Alka Arya and Shiv Prasad Yadav \\ Department of Mathematics, Indian Institute of Technology, Roorkee, India \\ e-mails: alkaldma@ittr.ac.in, yadavfma@ittr.ac.in
}

Received: 24 October $2017 \quad$ Revised: 2 December $2017 \quad$ Accepted: 4 December 2017

\begin{abstract}
Slack based measure (SBM) model determines the performance efficiency of homogeneous decision making units (DMUs) and also determines the efficient and inefficient DMUs. super-efficiency SBM (SESBM) model determines the efficiency of efficient DMUs. Guo et al. [7] proposed an integrated super-efficiency SBM (ISESBM) model which determines the efficiency and super-efficiency of DMUs using one model. In conventional ISESBM, the data is crisp. But it fluctuates in the real world applications. Such data can take the form of fuzzy / interval / intuitionistic fuzzy (IF) numbers. In this paper, we propose an IF ISESBM (IFISESBM) model using expected value of intuitionistic fuzzy numbers (IFNs). Finally, a health sector application of the proposed model is presented with two IF inputs and two IF outputs.
\end{abstract}

Keywords: Integrated super-efficiency slack based measure, Intuitionistic fuzzy integrated superefficiency slack based measure, Posterior efficiency.

2010 Mathematics Subject Classification: 03 E72.

\section{Introduction}

Slack based measure (SBM) model which is non-radial measure model based on slacks proposed by Tone et al. [12] determines the efficiencies, efficient and inefficient decision making units (DMUs). Tone et al. [13] proposed super-efficiency SBM (SESBM) model to determine the efficient DMUs. Du et al. [6] extended the SESBM model to additive SESBM model. In this model, firstly efficient DMUs are determined and then additive SESBM model is applied to determine the efficiencies of efficient DMUs. Guo et al. [7] proposed a model to determine the efficiencies and super efficiencies using one model which is known as integrated SESBM (ISESBM) model. 
Intuitionistic fuzzy set (IFS) theory proposed by Atanassov [2] is an extension of fuzzy set theory and have been found to be more useful to deal with vagueness/fluctuation. The IFS considers both the acceptance value and rejection value of an element such that the sum of both values is less than one, i.e., it may have hesitation. Since its invention/inception, the IFS theory has received more and more attention and has been used in a wide range of applications, such as, reliability [11], logic programming [3], decision making [8], medical diagnosis [4], pattern recognition [5]. Puri and Yadav [10] proposed IF optimistic-pessimistic DEA models to determine the efficiencies of DMUs in optimistic and pessimistic situations.

In this paper, community health centers (CHCs) have been taken to determine the efficiencies and slacks. Since the managers or other authorities reorganize facilities: non-medical staff and medical staff time to time, the fluctuation occurs in non-medical staff and medical staff, i.e., fluctuation in input data. Patients leave the hospital due to insufficient resources such as available beds for hospitalization and lackness to provide appropriate care etc. Hence fluctuation occurs in output data. Due to this, in order to deal with fluctuation in CHCs, the input data and output data are taken as intuitionistic fuzzy numbers (IFNs). So, fluctuation in input data and output data at hospital level can be well taken as IFN. In this paper, we extend ISESBM model to intuitionistic fuzzy ISESBM (IFISESBM) model using expected value of intuitionistic fuzzy numbers.

The rest of the paper is organized as follows: Section 2 presents preliminaries required to develop the model. Section 3 presents the proposed IFISESBM model. Section 4 presents an application to the health sector to illustrate the proposed model. Section 5 concludes the findings of this paper.

\section{Preliminaries}

\subsection{Intuitionistic fuzzy set (IFS)}

Let $\mathbb{X}$ be the universe of discourse. Then an IFS [2] is denoted by $\tilde{A}^{I}$ and defined by $\tilde{A}^{I}=$ $\left\{\left(x, \mu_{\tilde{A}^{I}}(x), \nu_{\tilde{A}^{I}}(x)\right)\right\}$, where $\mu_{\tilde{A}^{I}}: \mathbb{X} \rightarrow[0,1]$ and $\nu_{\tilde{A}^{I}}: \mathbb{X} \rightarrow[0,1]$ represent the membership and non-membership functions respectively. The values $\mu_{\tilde{A}^{I}}(x)$ and $\nu_{\tilde{A}^{I}}(x)$ represent the membership and non-membership values of $\mathrm{x}$ being in $\tilde{A}^{I}$ with the condition $0 \leq \mu_{\tilde{A}^{I}}(x)+\nu_{\tilde{A}^{I}}(x) \leq 1$, $\mu_{\tilde{A}^{I}}(x) \in[0,1]$ and $\nu_{\tilde{A}^{I}}(x) \in[0,1]$. The hesitation (indeterminacy) degree of an element $\mathrm{x}$ being in $\tilde{A}^{I}$ is defined as $\pi_{\tilde{A}^{I}}(x)=1-\mu_{\tilde{A}^{I}}(x)-\nu_{\tilde{A}^{I}}(x) \forall x \in \mathbb{X}$. Obviously $0 \leq \pi_{\tilde{A}^{I}}(x) \leq 1$. If $\pi_{\tilde{A}^{I}}(x)=0 \forall x \in \mathbb{X}$, then $\tilde{A}^{I}$ is reduced to a fuzzy set.

\subsection{Normal IFS}

Let $\tilde{A}^{I}=\left\{\left(x, \mu_{\tilde{A}^{I}}(x), \nu_{\tilde{A}^{I}}(x)\right) ; x \in \mathbb{X}\right\}$ be an IFS. Then $\tilde{A}^{I}$ is called normal IFS [2] if $\exists$ an $x \in \mathbb{X}$ such that $\mu_{\tilde{A}^{I}}(x)=1$ and $\nu_{\tilde{A}^{I}}(x)=0$.

\subsection{Convex IFS}

Let $\tilde{A}^{I}=\left\{\left(x, \mu_{\tilde{A}^{I}}(x), \nu_{\tilde{A}^{I}}(x)\right) ; x \in \mathbb{X}\right\}$ be an IFS. Then $\tilde{A}^{I}$ is called Convex IFS [2] if 
- $\min \left(\mu_{\tilde{A}^{I}}(x), \mu_{\tilde{A}^{I}}(y)\right) \leq \mu_{\tilde{A}^{I}}(\lambda x+(1-\lambda) y), \forall x, y \in \mathbb{X}$ and $\lambda \in[0,1]$, i.e., $\mu_{\tilde{A}^{I}}$ is quasiconcave over $\mathbb{X}$.

- $\max \left(\nu_{\tilde{A}^{I}}(x), \nu_{\tilde{A}^{I}}(y)\right) \geq \nu_{\tilde{A}^{I}}(\lambda x+(1-\lambda) y), \forall x, y \in \mathbb{X}$ and $\lambda \in[0,1]$, i.e., $\nu_{\tilde{A}^{I}}$ is quasiconvex over $\mathbb{X}$.

\subsection{Intuitionistic fuzzy number (IFN)}

Let $\tilde{A}^{I}=\left\{\left(x, \mu_{\tilde{A}^{I}}(x), \nu_{\tilde{A}^{I}}(x)\right): x \in \mathbb{R}\right\}$ be an IFS with its membership function $\mu_{\tilde{A}^{I}}$ and nonmembership function $\nu_{\tilde{A}^{I}}$, where $\mathbb{R}$ is the set of real numbers. Then $\tilde{A}^{I}$ is called an IFN [9] if the following conditions hold:

(i) $\exists$ a unique $x_{0} \in \mathbb{R}$ such that $\mu_{\tilde{A}^{I}}\left(x_{0}\right)=1$ and $\nu_{\tilde{A}^{I}}\left(x_{0}\right)=0$, i.e., $\tilde{A}^{I}$ is normal. $x_{0}$ is called the mean value of $\tilde{A}^{I}$.

(ii) $\tilde{A}^{I}$ is convex IFS.

Mathematically, an IFS $\tilde{A}^{I}=\left\{\left(x, \mu_{\tilde{A}^{I}}(x), \nu_{\tilde{A}^{I}}(x)\right): x \in \mathbb{R}\right\}$ is an IFN if $\mu_{\tilde{A}^{I}}$ and $\nu_{\tilde{A}^{I}}$ are piecewise continuous functions from $\mathbb{R}$ to $[0,1]$ and $0 \leq \mu_{\tilde{A}^{I}}(x)+\nu_{\tilde{A}^{I}}(x) \leq 1, \forall x \in \mathbb{R}$, where

$$
\mu_{\tilde{A}^{I}}(x)=\left\{\begin{array}{l}
g_{1}(x), a^{l} \leq x<a^{m}, \\
1, \quad x=a^{m}, \\
h_{1}(x), a^{m}<x \leq a^{u}, \\
0, \quad \text { elsewhere. }
\end{array} \quad \nu_{\tilde{A}^{I}}(x)=\left\{\begin{array}{l}
g_{2}(x), a^{l} \leq x<a^{m}, \\
0, \quad x=a^{m}, \\
h_{2}(x), a^{m}<x \leq a^{\prime u}, \\
1, \quad \text { elsewhere. }
\end{array}\right.\right.
$$

where $a^{m}$ is the mean value of $\tilde{A}^{I} ; a^{m}-a^{l}$ and $a^{u}-a^{m}$ are the left and right hand spreads of membership function $\mu_{\tilde{A}^{I}}$ respectively; $a^{m}-a^{l l}$ and $a^{\prime u}-a^{m}$ are the left and right hand spreads of hesitation function $\pi_{\tilde{A}^{I}}(x)$ respectively; $g_{1}$ and $h_{1}$ are piecewise continuous, strictly increasing and strictly decreasing functions in $\left[a^{l}, a^{m}\right)$ and $\left(a^{m}, a^{u}\right]$ respectively; $g_{2}$ and $h_{2}$ are piecewise continuous, strictly decreasing and strictly increasing functions in $\left[a^{\prime l}, a^{m}\right)$ and $\left(a^{m}, a^{\prime u}\right]$ respectively. Its graphical representation is given in Figure 1.

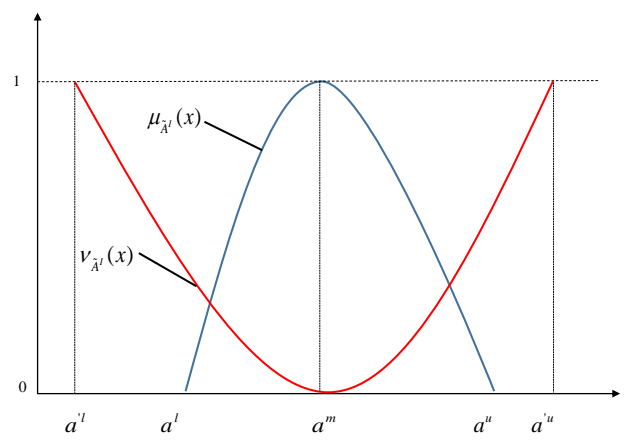

Figure 1: IFN

\subsection{Triangular intuitionistic fuzzy number (TIFN)}

TIFN [9] $\tilde{A}^{I}=\left(a^{l}, a^{m}, a^{u} ; a^{l l}, a^{m}, a^{\prime u}\right)$ is an IFN with the membership function $\mu_{\tilde{A}^{I}}$ and nonmembership function $\nu_{\tilde{A}^{I}}$ given by 


$$
\mu_{\tilde{A}^{I}}(x)=\left\{\begin{array}{l}
\frac{x-a^{l}}{a^{m}-a^{l}}, a^{l}<x \leq a^{m}, \\
\frac{a^{u}-x}{a^{u}-a^{m}}, a^{m} \leq x<a^{u} \\
0, \text { elsewhere. }
\end{array} \quad \nu_{\tilde{A}^{I}}(x)=\left\{\begin{array}{l}
\frac{x-a^{m}}{a^{\prime l}-a^{m}}, a^{\prime l}<x \leq a^{m}, \\
\frac{a^{m}-x}{a^{m}-a^{\prime u}}, a^{m} \leq x<a^{\prime u}, \\
1, \text { elsewhere. }
\end{array}\right.\right.
$$

where $a^{l}, a^{m}, a^{u}, a^{\prime l}, a^{\prime u} \in \mathbb{R}$ such that $a^{l} \leq a^{l} \leq a^{m} \leq a^{u} \leq a^{\prime u}$. Its graphical representation is given in Figure 2.

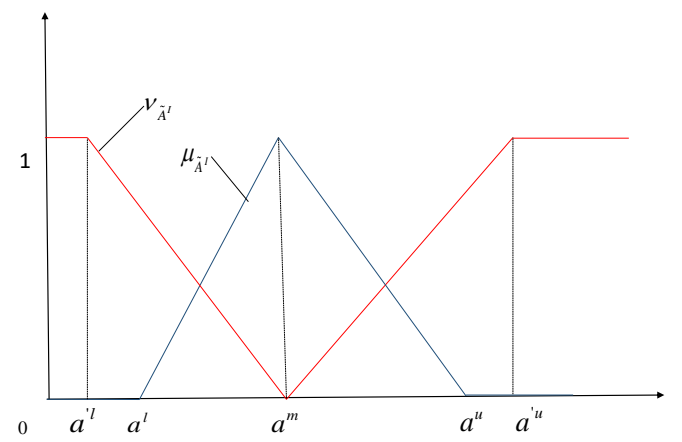

Figure 2: TIFN

\subsection{Expected interval (EI) of a TIFN}

The EI of TIFN $\tilde{A}^{I}=\left(a^{l}, a^{m}, a^{u} ; a^{\prime l}, a^{m}, a^{\prime u}\right)$ is defined as follows:

$E I\left(\tilde{A}^{I}\right)=\left[E^{L}\left(\tilde{A}^{I}\right), E^{U}\left(\tilde{A}^{I}\right)\right]$, where $E^{L}\left(\tilde{A}^{I}\right)=\frac{a^{l}+2 a^{m}+a^{\prime l}}{4}$ and $E^{U}\left(\tilde{A}^{I}\right)=\frac{a^{u}+2 a^{m}+a^{\prime u}}{4}$.

The expected value (EV) of a TIFN $\tilde{A}^{I}=\left(a^{l}, a^{m}, a^{u} ; a^{\prime l}, a^{m}, a^{\prime u}\right)$ is defined as follows: $E V\left(\tilde{A}^{I}\right)=\frac{1}{2}\left(E^{L}\left(\tilde{A}^{I}\right)+E^{U}\left(\tilde{A}^{I}\right)\right)=\frac{a^{l}+a^{\prime l}+4 a^{m}+a^{u}+a^{\prime u}}{8}$.

\section{Proposed intuitionistic fuzzy integrated}

\section{super-efficiency SBM (IFISESBM) model}

Guo et al. [7] proposed an integrated super-efficiency slack based measure (ISESBM) model. ISESBM is given in Model 1.

\section{Model 1}

$$
\begin{aligned}
& \min \xi_{j_{o}}=\sum_{i=1}^{m} t_{i j_{o}}^{+}+\sum_{r=1}^{s} t_{r j_{o}}^{-}-\varepsilon\left(\sum_{i=1}^{m} s_{i j_{o}}^{-}+\sum_{r=1}^{s} s_{r j_{o}}^{+}\right) \\
& \text {subject to } \sum_{j=1, \neq j_{o}}^{n n} x_{i j} \mu_{j_{o}}=x_{i j_{o}}+t_{i j_{o}}^{+}-s_{i j_{o}}^{-}, \\
& \sum_{j=1, \neq j_{o}}^{n} y_{r j} \mu_{j_{o}}=y_{r j_{o}}-t_{r j_{o}}^{-}+s_{r j_{o}}^{+}, \\
& \mu_{j_{o}} \geq 0, \forall j, \quad j \neq j_{o}, \\
& t_{i j_{o}}^{+} \geq 0, \forall i, \quad t_{r j_{o}}^{-} \geq 0, \forall r, \quad s_{i j_{o}}^{-} \geq 0, \forall i, \quad s_{r j_{o}}^{+} \geq 0, \forall r,
\end{aligned}
$$


where $s_{i j_{o}}^{-}$and $s_{r j_{o}}^{+}$are the inefficiency slacks, $t_{i j_{o}}^{+}$and $t_{r j_{o}}^{-}$are the super-efficiency slacks. In ISESBM model, firstly the super-efficiency slacks are determined and then inefficiency slacks are determined. Let $s_{i j_{o}}^{-*}, s_{r j_{o}}^{+*}, t_{i j_{o}}^{+*}$ and $t_{r j_{o}}^{-*}$ be the optimal values of $s_{i j_{o}}^{-}, s_{r j_{o}}^{+}, t_{i j_{o}}^{+}$and $t_{r j_{o}}^{-}$respectively. The posterior efficiency (PE) of Model 1 is defined as follows [7]

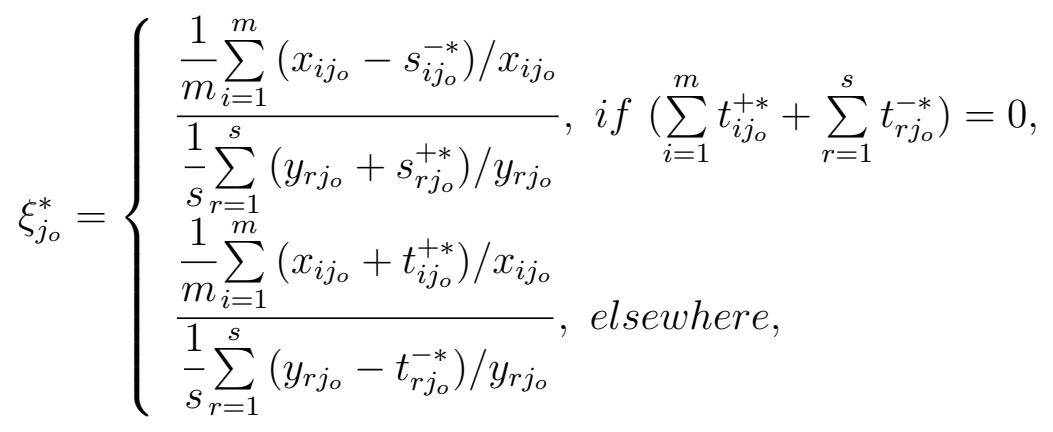

If $\xi_{j_{o}}^{*}>1$, then $D M U_{j_{o}}$ is ISESBM efficient and if $\xi_{j_{o}}^{*} \leq 1$, then $D M U_{j_{o}}$ is ISESBM inefficient [7].

\subsection{Intuitionistic fuzzy ISESBM model}

In conventional ISESBM the input data and output data are crisp values. But in the real world applications, these data may have intuitionistic fuzzy values [1]. Therefore, we have taken IF input-output data as TIFNs. Let $\tilde{x}_{i j}^{I}$ and $\tilde{y}_{r j}^{I}$ be the ith IF input and rth IF output respectively for $D M U_{j}$. Then we have the following model (Model 2)

\section{Model 2}

$$
\begin{aligned}
& \min \xi_{j_{o}}=\sum_{i=1}^{m} t_{i j_{o}}^{+}+\sum_{r=1}^{s} t_{r j_{o}}^{-}-\varepsilon\left(\sum_{i=1}^{m} s_{i j_{o}}^{-}+\sum_{r=1}^{s} s_{r j_{o}}^{+}\right) \\
& \text {subject to } \sum_{j=1, \neq j_{o}}^{n n} \tilde{x}_{i j}^{I} \mu_{j_{o}}=\tilde{x}_{i j_{o}}^{I}+t_{i j_{o}}^{+}-s_{i j_{o}}^{-} \\
& \sum_{j=1, \neq j_{o}}^{n} \tilde{y}_{r j}^{I} \mu_{j_{o}}=\tilde{y}_{r j_{o}}^{I}-t_{r j_{o}}^{-}+s_{r j_{o}}^{+}, \\
& \mu_{j_{o}} \geq 0, \forall j, \quad j \neq j_{o}, \\
& t_{i j_{o}}^{+} \geq 0, \forall i, \quad t_{r j_{o}}^{-} \geq 0, \forall r, \quad s_{i j_{o}}^{-} \geq 0, \forall i, \quad s_{r j_{o}}^{+} \geq 0, \forall r .
\end{aligned}
$$

The posterior efficiency (PE) of Model 2 is defined as follows

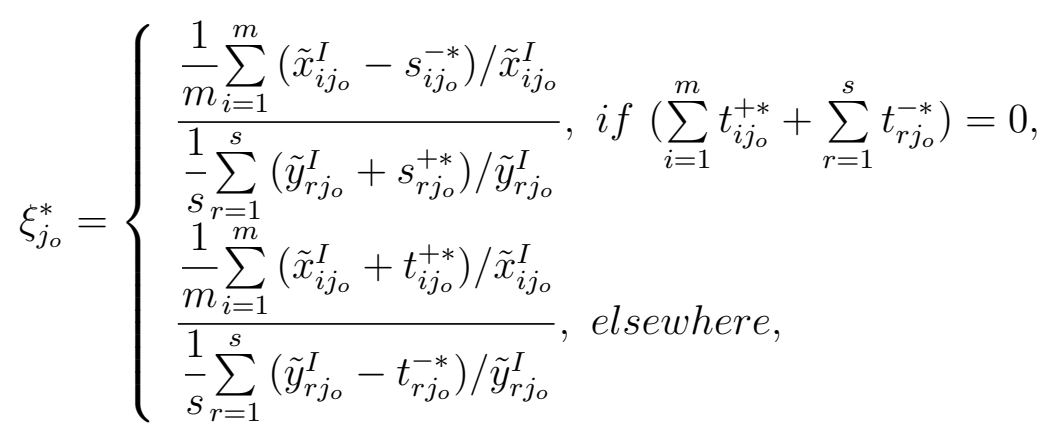


Let the IF input and IF output be TIFNs:

$$
\begin{gathered}
\left(\tilde{x}_{i j}^{I}\right)=\left(x_{i j}^{l}, x_{i j}^{m}, x_{i j}^{u} ; x_{i j}^{\prime}, x_{i j}^{m}, x_{i j}^{\prime u}\right), \\
\left(\tilde{y}_{r j}^{I}\right)=\left(y_{r j}^{l}, y_{r j}^{m}, y_{r j}^{u} ; y_{r j}^{\prime l}, y_{r j}^{m}, y_{r j}^{\prime}\right), \\
\left(\tilde{x}_{i j_{o}}^{I}\right)=\left(x_{i j_{o}}^{l}, x_{i j_{o}}^{m}, x_{i j_{o}}^{u} ; x_{i j_{o}}^{l}, x_{i j_{o}}^{m}, x_{i j_{o}}^{\prime}\right)
\end{gathered}
$$

and

$$
\left(\tilde{y}_{r j_{o}}^{I}\right)=\left(y_{r j_{o}}^{l}, y_{r j_{o}}^{m}, y_{r j_{o}}^{u} ; y_{r j_{o}}^{\prime l}, y_{r j_{o}}^{m}, y_{r j_{o}}^{\prime}\right)
$$

Then we get Model 3.

\section{Model 3}

$\min \xi_{j_{o}}=\sum_{i=1}^{m} t_{i j_{o}}^{+}+\sum_{r=1}^{s} t_{r j_{o}}^{-}-\varepsilon\left(\sum_{i=1}^{m} s_{i j_{o}}^{-}+\sum_{r=1}^{s} s_{r j_{o}}^{+}\right)$

subject to $\sum_{j=1, \neq j_{o}}^{n n}\left(x_{i j}^{l}, x_{i j}^{m}, x_{i j}^{u} ; x_{i j}^{\prime l}, x_{i j}^{m}, x_{i j}^{\prime} u\right) \mu_{j_{o}}=\left(x_{i j_{o}}^{l}, x_{i j_{o}}^{m}, x_{i j_{o}}^{u} ; x_{i j_{o}}^{\prime l}, x_{i j_{o}}^{m}, x_{i j_{o}}^{\prime u}\right)+t_{i j_{o}}^{+}-s_{i j_{o}}^{-}$,

$\sum_{j=1, \neq j_{o}}^{n}\left(y_{r j}^{l}, y_{r j}^{m}, y_{r j}^{u} ; y_{r j}^{\prime l}, y_{r j}^{m}, y_{r j}^{\prime u}\right) \mu_{j_{o}}=\left(y_{r j_{o}}^{l}, y_{r j_{o}}^{m}, y_{r j_{o}}^{u} ; y_{r j_{o}}^{\prime l}, y_{r j_{o}}^{m}, y_{r j_{o}}^{\prime u}\right)-t_{r j_{o}}^{-}+s_{r j_{o}}^{+}$,

$\mu_{j_{o}} \geq 0, \forall j, j \neq j_{o}$,

$t_{i j_{o}}^{+} \geq 0, \forall i, t_{r j_{o}}^{-} \geq 0, \forall r, s_{i j_{o}}^{-} \geq 0, \forall i, s_{r j_{o}}^{+} \geq 0, \forall r$.

Taking EV of TIFNs, we get Model 4 from Model 3.

\section{Model 4}

$\min E V\left(\xi_{j_{o}}\right)=\sum_{i=1}^{m} t_{i j_{o}}^{+}+\sum_{r=1}^{s} t_{r j_{o}}^{-}-\varepsilon\left(\sum_{i=1}^{m} s_{i j_{o}}^{-}+\sum_{r=1}^{s} s_{r j_{o}}^{+}\right)$

subject to

$$
\begin{aligned}
& \sum_{j=1, \neq j_{o}}^{n n} E V\left(x_{i j}^{l}, x_{i j}^{m}, x_{i j}^{u} ; x_{i j}^{\prime l}, x_{i j}^{m}, x_{i j}^{\prime u}\right) \mu_{j_{o}}=E V\left(x_{i j_{o}}^{l}, x_{i j_{o}}^{m}, x_{i j_{o}}^{u} ; x_{i j_{o}}^{\prime l}, x_{i j_{o}}^{m}, x_{i j_{o}}^{\prime}\right)+t_{i j_{o}}^{+}-s_{i j_{o}}^{-}, \\
& \sum_{j=1, \neq j_{o}}^{n} E V\left(y_{r j}^{l}, y_{r j}^{m}, y_{r j}^{u} ; y_{r j}^{\prime l}, y_{r j}^{m}, y_{r j}^{\prime u}\right) \mu_{j_{o}}=E V\left(y_{r j_{o}}^{l}, y_{r j_{o}}^{m}, y_{r j_{o}}^{u} ; y_{r j_{o}}^{\prime l}, y_{r j_{o}}^{m}, y_{r j_{o}}^{\prime}\right)-t_{r j_{o}}^{-}+s_{r j_{o}}^{+}, \\
& \mu_{j_{o}} \geq 0, \forall j, \quad j \neq j_{o}, \\
& t_{i j_{o}}^{+} \geq 0, \forall i, \quad t_{r j_{o}}^{-} \geq 0, \forall r, \quad s_{i j_{o}}^{-} \geq 0, \forall i, s_{r j_{o}}^{+} \geq 0, \forall r .
\end{aligned}
$$

We get Model 5 from Model 4 using expected value of TIFNs, which is given in Definition 7. 


\section{Model 5}

$\min E_{j_{o}}=\sum_{i=1}^{m} t_{i j_{o}}^{+}+\sum_{r=1}^{s} t_{r j_{o}}^{-}-\varepsilon\left(\sum_{i=1}^{m} s_{i j_{o}}^{-}+\sum_{r=1}^{s} s_{r j_{o}}^{+}\right)$

subject to

$$
\begin{aligned}
& \sum_{j=1, \neq j_{o}}^{n}\left(x_{i j}^{l}+4 x_{i j}^{m}+x_{i j}^{u}+x_{i j}^{\prime l}+x_{i j}^{\prime u}\right) \mu_{j_{o}}=\left(x_{i j_{o}}^{l}+4 x_{i j_{o}}^{m}+x_{i j_{o}}^{u}+x_{i j_{o}}^{\prime l}+x_{i j_{o}}^{\prime u}\right)+8 t_{i j_{o}}^{+}-8 s_{i j_{o}}^{-}, \\
& \sum_{j=1, \neq j_{o}}^{n}\left(y_{r j}^{l}+4 y_{r j}^{m}+y_{r j}^{u}+y_{r j}^{\prime l}+y_{r j}^{\prime}\right) \mu_{j_{o}}=\left(y_{r j_{o}}^{l}+4 y_{r j_{o}}^{m}+y_{r j_{o}}^{u}+y_{r j_{o}}^{\prime l}+y_{r j_{o}}^{\prime} u-8 t_{r j_{o}}^{-}+8 s_{r j_{o}}^{+},\right. \\
& \mu_{j_{o}} \geq 0, \forall j, \quad j \neq j_{o}, \\
& t_{i j_{o}}^{+} \geq 0, \forall i, \quad t_{r j_{o}}^{-} \geq 0, \forall r, \quad s_{i j_{o}}^{-} \geq 0, \forall i, \quad s_{r j_{o}}^{+} \geq 0, \forall r .
\end{aligned}
$$

Model 5 is the proposed intuitionistic fuzzy ISESBM (PIFISESBM) model. The posterior efficiency (PE) of PIFISESBM is defined as follows

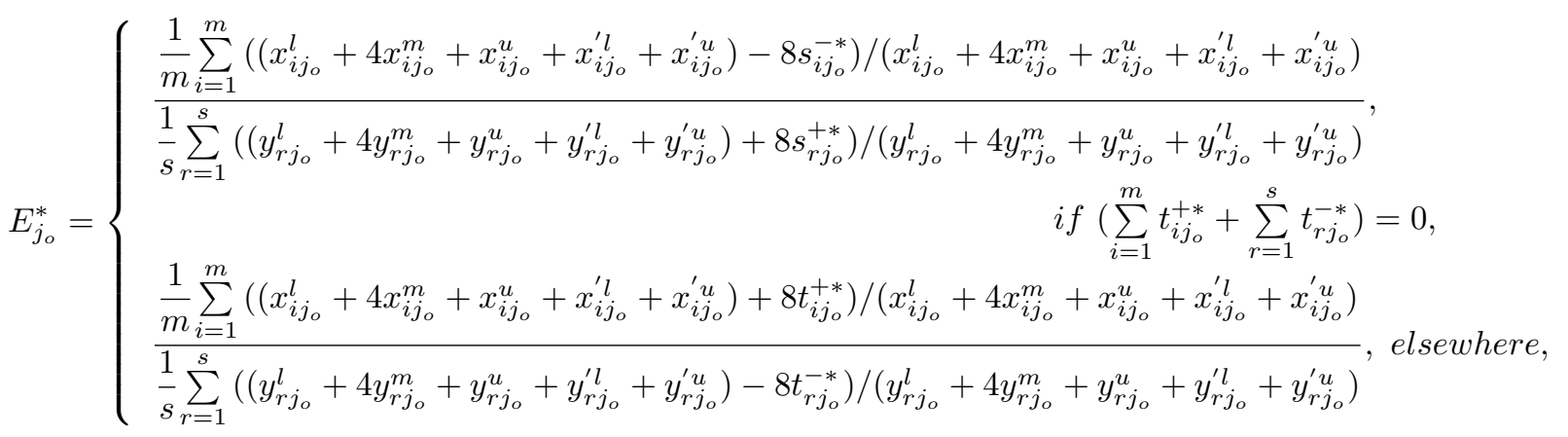

If $E_{j_{o}}^{*}>1$, then $D M U_{j_{o}}$ is PIFISESBM efficient and if $E_{j_{o}}^{*} \leq 1$, then $D M U_{j_{o}}$ is PIFISESBM inefficient.

\section{Real life application}

Let us consider a real life application to illustrate the proposed model. Let there be two IF inputs: (i) number of doctors $=\tilde{x}_{1 j}^{I}$, (ii) number of pharmacists $=\tilde{x}_{2 j}^{I}$, and two fuzzy outputs: (i) number of inpatients $=\tilde{y}_{1 j}^{I}$, (ii) number of outpatients $=\tilde{y}_{2 j}^{I}$ of community health centers (CHCs) in Meerut district of Uttar Pradesh, India and IF input data and output of CHCs are shown in Tables 1 and 2, respectively, where the CHC codes respectively stay for: $\mathrm{H} 1 \rightarrow$ Mawana, $\mathrm{H} 2 \rightarrow$ Sardhana, H3 $\rightarrow$ Daurala, H4 $\rightarrow$ Bhudbharal, H5 $\rightarrow$ Jani, H6 $\rightarrow$ Rohta, H7 $\rightarrow$ Kharkhoda, H8 $\rightarrow$ Hastinapur, H9 $\rightarrow$ P.Garh, H10 $\rightarrow$ Bhavanpur, H11 $\rightarrow$ Machra, H12 $\rightarrow$ S.Khurd.

The inefficiency slacks $\left(s_{1 j}^{-}, s_{2 j}^{-}, s_{1 j}^{+}\right.$and $\left.s_{2 j}^{+}\right)$and super-efficiency slacks $\left(t_{1 j}^{+}, t_{2 j}^{+}, t_{1 j}^{-}\right.$and $t_{2 j}^{-}$) of each CHC are determined using PIFISESBM model ( $\varepsilon$ is taken as $\left.10^{-4}\right)$ and are shown in Table 3. In Table 3, $s_{1 j}^{-}$and $s_{2 j}^{-}$are input inefficiency slacks corresponding to $\tilde{x}_{1 j}^{I}$ and $\tilde{x}_{2 j}^{I}$ respectively; $s_{1 j}^{+}$and $s_{2 j}^{+}$are output inefficiency slacks corresponding to $\tilde{y}_{1 j}^{I}$ and $\tilde{y}_{2 j}^{I}$ respectively; $t_{1 j}^{+}$and $t_{2 j}^{+}$are input super-efficiency slacks corresponding to $\tilde{x}_{1 j}^{I}$ and $\tilde{x}_{2 j}^{I} ; t_{1 j}^{-}$and $t_{2 j}^{-}$are output super-efficiency slacks corresponding to $\tilde{y}_{1 j}^{I}$ and $\tilde{y}_{2 j}^{I}$ respectively. The posterior efficiency, $E_{j}^{*}$, of each CHC is determined and is shown in Table 3. $H 2, H 3, H 4, H 5, H 6, H 7, H 10, H 11, H 12$ 
CHCs are PIFISESBM efficient; other CHCs are PIFISESBM inefficient. Rank of the CHCs is $H 4>H 6>H 3>H 2>H 11>H 7>H 10>H 5>H 12>H 1 \geq H 8 \geq H 9$.

Table 1: IF input data of 12 hospitals

\begin{tabular}{|c|c|c|}
\hline$\overline{\mathrm{DMU}}$ & \multicolumn{2}{|c|}{ IF inputs } \\
\hline & $\tilde{x}_{1 j}^{I}$ & $\tilde{x}_{2 j}^{I}$ \\
\hline $\mathrm{H} 1$ & $(5,10,19 ; 3,10,28)$ & $(3,5,8 ; 2,5,10)$ \\
\hline $\mathrm{H} 2$ & $(4,9,17 ; 3,9,26)$ & $(3,5,7 ; 2,5,9)$ \\
\hline $\mathrm{H} 3$ & $(8,11,19 ; 5,11,29)$ & $(2,4,5 ; 1,4,6)$ \\
\hline $\mathrm{H} 4$ & $(3,8,15 ; 2,8,22)$ & $(1,1,3 ; 1,1,5)$ \\
\hline H5 & $(6,10,18 ; 5,10,26)$ & $(3,4,6 ; 2,4,10)$ \\
\hline H6 & $(7,11,21 ; 5,11,31)$ & $(2,3,4 ; 1,3,5)$ \\
\hline $\mathrm{H} 7$ & $(8,10,16 ; 5,10,25)$ & $(1,2,6 ; 1,2,18)$ \\
\hline $\mathrm{H} 8$ & $(7,11,18 ; 5,11,29)$ & $(2,4,7 ; 1,4,19)$ \\
\hline H9 & $(8,12,19 ; 5,12,28)$ & $(2,5,7 ; 1,5,15)$ \\
\hline $\mathrm{H} 10$ & $(9,15,19 ; 8,15,22)$ & $(2,4,6 ; 1,4,18)$ \\
\hline H11 & $(6,11,16 ; 5,11,18)$ & $(3,5,8 ; 2,5,20)$ \\
\hline $\mathrm{H} 12$ & $(4,8,11 ; 3,8,14)$ & $(3,4,6 ; 1,4,7)$ \\
\hline
\end{tabular}

Table 2: IF output data of 12 hospitals

\begin{tabular}{ccc}
\hline DMU & \multicolumn{2}{c}{ IF outputs } \\
\hline & $\tilde{y}_{1 j}^{I}$ & $\tilde{y}_{2 j}^{I}$ \\
\hline H1 & $(3640,3650,3665 ; 3635,3650,3695)$ & $(134130,134137,134140 ; 134125,134137,134145)$ \\
H2 & $(4150,4160,4175 ; 4148,4160,4195)$ & $(116060,116062,116070 ; 116055,116062,116075)$ \\
H3 & $(4360,4370,4380 ; 4357,4370,4398)$ & $(94060,94066,94070 ; 94055,94066,94075)$ \\
H4 & $(485,492,500 ; 483,492,515)$ & $(24325,24329,24334 ; 24322,24329,24338)$ \\
H5 & $(2460,2464,2470 ; 2458,2464,2475)$ & $(99745,99748,99750 ; 99742,99748,99755)$ \\
H6 & $(1360,1368,1378 ; 1358,1368,1398)$ & $(49398,49401,49405 ; 49395,49401,49409)$ \\
H7 & $(1055,1062,1080 ; 1050,1062,1083)$ & $(37769,37772,37776 ; 37765,37772,37779)$ \\
H8 & $(1295,1302,1310 ; 1290,1302,1325)$ & $(82838,82841,82845 ; 82835,82841,82849)$ \\
H9 & $(1660,1671,1690 ; 1657,1671,16105)$ & $(100590,100596,100600 ; 100586,100596,100605)$ \\
H10 & $(1010,1018,1035 ; 1008,1018,1045)$ & $(64349,64351,64358 ; 64345,64351,64360)$ \\
H11 & $(1500,1504,1515 ; 1495,1504,1535)$ & $(80050,80056,80060 ; 80045,80056,80065)$ \\
H12 & $(1960,1965,1972 ; 1958,1965,1985)$ & $(58160,58167,58170 ; 58157,58167,58174)$ \\
\hline
\end{tabular}


Table 3: Input-output slacks and efficiencies

\begin{tabular}{cccccccccc}
\hline DMUs & \multicolumn{3}{c}{ Input slacks } & \multicolumn{2}{c}{ Output slacks } & \multicolumn{3}{c}{ Input slacks } & \multicolumn{2}{c}{ Output slacks } & Efficiency \\
\hline & $s_{1 j}^{-}$ & $s_{2 j}^{-}$ & $s_{1 j}^{+}$ & $s_{2 j}^{+}$ & $t_{1 j}^{+}$ & $t_{2 j}^{+}$ & $t_{1 j}^{-}$ & $t_{2 j}^{-}$ & $E_{j}^{*}$ \\
\hline H1 & 0 & 0 & 1157.43 & 0 & 0.548 & 0.548 & 0 & 0 & 1.0 \\
H2 & 0 & 0 & 0 & 3676.23 & 2.779 & 0.99 & 0 & 0 & 1.2 \\
H3 & 0 & 0 & 0 & 52102.48 & 0 & 2.29 & 0 & 0 & 1.3 \\
H4 & 0 & 0 & 2352.69 & 80155.51 & 0 & 2.436 & 0 & 0 & 1.7 \\
H5 & 0 & 0 & 1189.5 & 34388 & 0 & 0.75 & 0 & 0 & 1.1 \\
H6 & 0 & 0 & 2783.69 & 103090.1 & 0 & 3.11 & 0 & 0 & 1.5 \\
H7 & 0 & 0 & 2551.4 & 94951.92 & 0 & 1.068 & 0 & 0 & 1.13 \\
H8 & 0 & 0 & 2658.61 & 62590.29 & 0 & 0.202 & 0 & 0 & 1.0 \\
H9 & 0 & 0 & 679.94 & 51895.83 & 0 & 0.485 & 0 & 0 & 1.0 \\
H10 & 0 & 0 & 3517.87 & 102259 & 0 & 1.3 & 0 & 0 & 1.12 \\
H11 & 0 & 0 & 2996.6 & 85274.9 & 3.511 & 0 & 0 & 0 & 1.16 \\
H12 & 0 & 0 & 837.64 & 44775.46 & 1.1133 & 0 & 0 & 0 & 1.06 \\
\hline
\end{tabular}

\section{Conclusion}

The real world applications data have some degrees of fluctuations. To deal with such data, we have considered them as TIFNs. In this paper, we extended integrated super-efficiency SBM model to intuitionistic fuzzy integrated super effciency SBM (IFISESBM) model. IFISESBM model determines the efficiencies and super efficiencies of DMUs using one model in IF environment. To ensure the validity of the proposed models, we have considered the performance of CHCs with two IF inputs and two IF outputs (Tables 1 and 2). PIFISESBM model is more effective for real world applications. PIFISESBM model also determines the efficient and inefficient CHCs. These efficiencies and input-output slacks provide extra information to the decision maker. This paper has some limitations. The proposed models are studied under the SBM model. The uncertainty in this paper is limited to TIFNs. We plan to extend these models to the other DEA models and also plan to use the trapezoidal IFNs and interval valued intuitionistic fuzzy sets to determine the efficiencies of real world applications.

\section{Acknowledgement}

The authors are thankful to the Ministry of Human Resource Development (MHRD), Govt. of India, India for financial assistance. The authors are also thankful to Mr. Deen Bandhu, ARO, Chief Medical Office, Meerut, India for providing the valuable input-output data of CHCs. 


\section{References}

[1] Arya, A., \& Yadav, S. P. (2017) A fuzzy dual SBM model with fuzzy weights: An application to the health sector, Proceedings of Sixth International Conference on Soft Computing for Problem Solving, Springer, pp. 230-238.

[2] Atanassov, K. T. (1986) Intuitionistic fuzzy sets, Fuzzy Sets and Systems, 20(1), 87-96.

[3] Atanassov, K. T. (1999) Elements of intuitionistic fuzzy logics, Intuitionistic Fuzzy Sets: Theory and Applications, Springer, pp. 199-236.

[4] De, S. K., Biswas, R., \& Roy, A. R. (2001) An application of intuitionistic fuzzy sets in medical diagnosis, Fuzzy Sets and Systems, 117(2), 209-213.

[5] Dengfeng, L., \& Chuntian, C. (2002) New similarity measures of intuitionistic fuzzy sets and application to pattern recognitions, Pattern Recognition Letters, 23(1), 221-225.

[6] Du, J., Liang, L., \& Zhu, J. (2010) A slacks-based measure of super-efficiency in data envelopment analysis: a comment, European Journal of Operational Research, 204(3), 694-697.

[7] Guo, I. L., Lee, H. S., \& Lee, D. (2017) An integrated model for slack-based measure of super-efficiency in additive DEA, Omega, 67, 160-167.

[8] Li, D. F. (2005) Multiattribute decision making models and methods using intuitionisticfuzzy sets, Journal of Computer and System Sciences, 70(1), 73-85.

[9] Mahapatra, G. S., \& Roy, T. K. (2009) Reliability evaluation using triangular intuitionistic fuzzy numbers arithmetic operations, World Academy of Science, Engineering and Technology, 50, 574-581.

[10] Puri, J., \& Yadav, S. P. (2015) Intuitionistic fuzzy data envelopment analysis: An application to the banking sector in India, Expert Systems with Applications, 42(11), 4982-4998.

[11] Shu, M. H., Cheng, C. H., \& Chang, J. R. (2006) Using intuitionistic fuzzy sets for fault-tree analysis on printed circuit board assembly. Microelectronics Reliability, 46(12), 2139-2148.

[12] Tone, K. (2001) A slacks-based measure of efficiency in data envelopment analysis, European Journal of Operational Research, 130(3), 498-509.

[13] Tone, K. (2002) A slacks-based measure of super-efficiency in data envelopment analysis, European Journal of Operational Research, 143(1), 32-41. 